\title{
Vacancy defect and defect cluster energetics in ion-implanted $\mathrm{ZnO}$
}

\author{
Yufeng Dong, ${ }^{1, *}$ F. Tuomisto, ${ }^{2}$ B. G. Svensson, ${ }^{3}$ A. Yu. Kuznetsov, ${ }^{3}$ and Leonard J. Brillson ${ }^{1,4,5}$ \\ ${ }^{1}$ Department of Electrical and Computer Engineering, The Ohio State University, Columbus, Ohio 43210, USA \\ ${ }^{2}$ Department of Applied Physics, Helsinki University of Technology, P.O. Box 1100, Helsinki 02015 TKK, Finland \\ ${ }^{3}$ Department of Physics, University of Oslo, P.O. Box 1048, Blindern, 0316 Oslo, Norway \\ ${ }^{4}$ Department of Physics, The Ohio State University, Columbus, Ohio 43210, USA \\ ${ }^{5}$ Center for Materials Research, The Ohio State University, Columbus, Ohio 43210, USA
}

(Received 8 January 2010; published 1 February 2010)

\begin{abstract}
We have used depth-resolved cathodoluminescence, positron annihilation, and surface photovoltage spectroscopies to determine the energy levels of $\mathrm{Zn}$ vacancies and vacancy clusters in bulk $\mathrm{ZnO}$ crystals. Doppler broadening-measured transformation of $\mathrm{Zn}$ vacancies to vacancy clusters with annealing shifts defect energies significantly lower in the $\mathrm{ZnO}$ band gap. $\mathrm{Zn}$ and corresponding $\mathrm{O}$ vacancy-related depth distributions provide a consistent explanation of depth-dependent resistivity and carrier-concentration changes induced by ion implantation.
\end{abstract}

\section{DOI: 10.1103/PhysRevB.81.081201}

$\mathrm{ZnO}$ is a leading candidate for next generation optoelectronic materials because of its large band gap, high exciton binding energy, thermochemical stability, and environmental compatibility. ${ }^{1,2}$ High quality single-crystal bulk $\mathrm{ZnO}$ wafers grown by various methods are commercially available ${ }^{3}$ and $\mathrm{ZnO}$ thin-film growth has attracted intense interest. ${ }^{4}$ However, despite nearly sixty years of research, several fundamental issues surrounding $\mathrm{ZnO}$ remain unresolved. Chief among these have been the difficulty of $p$-type doping and the role of compensating native defects. ${ }^{5,6}$ Oxygen vacancies $\left(V_{\mathrm{O}}\right), V_{\mathrm{O}}$ complexes, $\mathrm{Zn}$ interstitial-related complexes, and residual impurities such as hydrogen and aluminum are all believed to be shallow donors in $\mathrm{ZnO}$, while $\mathrm{Zn}$ vacancies $\left(V_{\mathrm{Zn}}\right)$ and their complexes are considered to be acceptors. ${ }^{7,8}$ Although their impact on carrier compensation is recognized, the physical nature of the donors and acceptors dominating carrier densities in $\mathrm{ZnO}$ is unresolved. Thus it remains a challenge to correlate the commonly observed $1.9-2.1 \mathrm{eV}$ "red" and 2.3-2.5 eV "green" luminescence emissions with specific native defects. 9 These and other emissions vary widely in $\mathrm{ZnO}$ bulk or thin films grown by various methods. ${ }^{10-14}$ Previous optical absorption, photoluminescence, electron paramagnetic resonance, and depth-resolved cathodoluminescence spectroscopy (DRCLS) (Ref. 15) studies indicate a correlation between the "green" optical transition and $\mathrm{O}$ vacancies $\left(V_{\mathrm{O}}\right) \cdot{ }^{10,16}$ Still controversial, however, is how such visible emissions correlate with the energetics of $\mathrm{Zn} / \mathrm{O}$ vacancies, interstitials, and their complexes overall. This work clearly identifies the physical nature of the defects dominating optical features of this widely studied semiconductor and, in turn, these defects provide a consistent explanation for ZnO's effective free-carrier densities on a local scale.

Contemporary theoretical approaches are also limited in addressing $\mathrm{ZnO}$ defect energetics due to major uncertainties, most notably, the "band-gap problem" within densityfunctional methods. ${ }^{17}$ Calculations of such basic $\mathrm{ZnO}$ defect properties as formation energy and energy-level relative to band edges vary considerably with different approximations. ${ }^{5,18-21}$ Therefore, the determination of energy levels of native point defects and energetics of $\mathrm{Zn}$ vacancies versus their clusters provides a method to evaluate methods
PACS number(s): 72.40.+w, 71.55.Gs, 78.60.Hk

for calculating deep level energies within $\mathrm{ZnO}$ and other semiconductors.

Here we augment the depth-resolved luminescence of energy-level transitions involving native defects with recent positron-annihilation spectroscopy (PAS) results ${ }^{22,23}$ to determine the energetics of $V_{\mathrm{Zn}}$ and their complexes in $\mathrm{ZnO}$ over both surface and near-surface regions $(7 \sim 1500 \mathrm{~nm})$ in ion ( $\mathrm{Li}$ or $\mathrm{N}$ ) implanted and annealed bulk $\mathrm{ZnO}$. Both $\mathrm{Li}$ and $\mathrm{N}$ are among the most important dopants for $p$-type $\mathrm{ZnO}$ doping, yet the roles of the associated defects generated by implantation or annealing are not yet clear. Doppler broadening experiments with a slow positron beam provide depth distributions of neutral or negatively charged vacancy defects, ${ }^{24,25}$ in this case, of $V_{\mathrm{Zn}}$ and vacancy clusters. The correspondence between these PAS native defect distributions and the DRCLS intensity distributions versus depth permits us to identify the luminescence energy associated with isolated $V_{\mathrm{Zn}}$ defects as well as the energy shift due to vacancy cluster formation. Surface photovoltage spectroscopy (SPS) yields the positions of these levels with respect to the $\mathrm{ZnO}$ band edges. We associate the remaining deep level DRCLS emission with positively charged $V_{\mathrm{O}}$-related defects, which are not detected by PAS, and describe how the balance between these donor and acceptor defects accounts for depthdependent resistivity in these irradiated crystals. Taking these depth-resolved techniques altogether, we clearly identify the optical transitions and energies of $V_{\mathrm{Zn}}$ and vacancy clusters, the effects of different annealing methods on their spatial distributions in ion-implanted $\mathrm{ZnO}$, and the contribution of $V_{\mathrm{Zn}}$ and $V_{\mathrm{O}}$ to near-surface resistivity.

In order to create well-defined distributions of $\mathrm{Zn}$ vacancies, we implanted (0001) $\mathrm{ZnO}$ wafers with ${ }^{7} \mathrm{Li}^{+}$or ${ }^{14} \mathrm{~N}^{+}$and annealed by conventional furnace or flash lamp at various temperatures. These crystals were hydrothermally grown, unintentionally doped with $\sim 5 \times 10^{17} \mathrm{Li} / \mathrm{cm}^{3}, n$ type and highly resistive. The wafers were annealed by conventional furnace or flash lamp at various temperatures. Details of these samples and their preparation appear elsewhere. ${ }^{22,23}$ Electron beams with incident energy $E_{\mathrm{B}}=1,2,3,4$, and 5 $\mathrm{keV}$ excited electron-hole pairs for peak DRCLS excitation depth $U_{0}=7,18,32,50$, and $72 \mathrm{~nm}$, respectively for specimens at $70 \mathrm{~K}$ in UHV. DRCLS with higher $E_{\mathrm{B}}(5-25 \mathrm{keV})$ at 


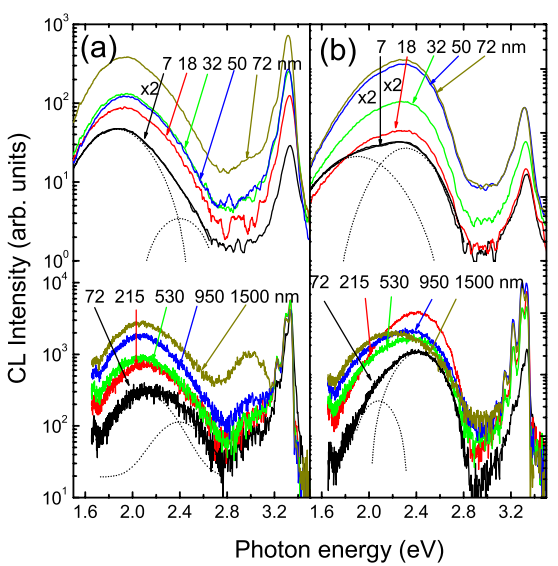

FIG. 1. (Color online) 70 (1-5) and $10 \mathrm{~K}(5-25 \mathrm{keV}) \mathrm{CL}$ spectra for Li-implanted $\mathrm{ZnO}$ after (a) flash anneal at $1200{ }^{\circ} \mathrm{C}$ and (b) furnace anneal at $800{ }^{\circ} \mathrm{C}$. Dashed lines represent characteristic emissions at $\sim 2.0$ and $\sim 2.4 \mathrm{eV}$ as revealed by fitting.

$10 \mathrm{~K}$ employed a JEOL 7800F UHV scanning electron microprobe with hemispherical electron analyzer and Oxford optical train. For $E_{\mathrm{B}}=10,15,20$, and $25 \mathrm{keV}$, Monte Carlo simulations including backscattering produce $U_{0}=215,530$, 950, and $1500 \mathrm{~nm}$, respectively. A two-Gaussian peak fit to the DRCLS spectra provided characteristic defect intensities $I_{\mathrm{D}}$ at nominally $\sim 2.0$ and $\sim 2.4 \mathrm{eV}$. Near band-edge (NBE) intensities at $3.4 \mathrm{eV}$ were corrected for attenuation due to self absorption ${ }^{26}$ at bulk depths $\left(E_{\mathrm{B}}>5 \mathrm{keV}\right)$.

For Li-implanted $\mathrm{ZnO}$, PAS depth profiles of the $S$ parameter extracted from Doppler broadening spectra ${ }^{22}$ show: (i) an increase in the concentration of open volume defects after implantation, (ii) formation of vacancy clusters with open volume larger than that of single $V_{\mathrm{Zn}}$ after flash $(20 \mathrm{~ms})$ annealing at $1200{ }^{\circ} \mathrm{C}$, and (iii) disappearance of these clusters after conventional furnace annealing for $1 \mathrm{~h}$ at $800{ }^{\circ} \mathrm{C}$. DRCLS spectra of the as-implanted $\mathrm{ZnO}$ (not shown) displays broad deep level emissions extending from $<1.9-2.5 \mathrm{eV}$ with peak defect intensity $I_{\mathrm{D}}$ normalized to $I_{\mathrm{NBE}}$ such that $I_{\mathrm{D}}(\sim 2.0 \mathrm{eV}) / I_{\mathrm{NBE}}(5 \mathrm{keV})$ and $I_{\mathrm{D}}(\sim 2.4 \mathrm{eV}) / I_{\mathrm{NBE}}(5 \mathrm{keV})=0.08$ and 0.035 , respectively. In contrast to conventional furnace anneals, flash anneals of $\mathrm{Li}-$ implanted $\mathrm{ZnO}$ generates stable and electrically active $V_{\mathrm{Zn}}$ clusters. Figures 1(a) and 1(b) show the spectra for Liimplanted samples after fast $(20 \mathrm{~ms})$ flash $\left(\right.$ at $\left.1200{ }^{\circ} \mathrm{C}\right)$ and $1 \mathrm{~h}$ furnace (at $800{ }^{\circ} \mathrm{C}$ ) annealing respectively. Besides $\sim 2.0, \sim 2.4$, and $3.4 \mathrm{eV}$ features, phonon replicas appear below the band edge in the $10 \mathrm{~K}$ spectra. A $3 \mathrm{eV}$ bulk emission evident in Fig. 1(a) is removed by furnace anneal in Fig. 1(b). Flash-annealed $I_{\mathrm{D}}(\sim 2.0 \mathrm{eV}) / I_{\mathrm{NBE}}(5 \mathrm{keV})=0.57$ near the surface, nearly unchanged (0.48) in the bulk $(25 \mathrm{keV})$, whereas furnace-annealed $I_{\mathrm{D}}(\sim 2.0 \mathrm{eV}) / I_{\mathrm{NBE}}(5 \mathrm{keV})=3$ near the surface decreasing to 0.16 in the bulk. Furthermore, flash-annealed $I_{\mathrm{D}}(\sim 2.4 \mathrm{eV}) / I_{\mathrm{NBE}}$ is low $(0.17)$ for both surface and bulk, whereas it increases 35 times at the surface and decreases 1.7 times in the bulk after furnace annealing. Lower-temperature $\left(500{ }^{\circ} \mathrm{C}\right)$ furnace anneals produce relatively few changes. Thus higher-temperature flash and furnace annealing produce major changes in the depth distributions of both $\sim 2.0$ and $\sim 2.4 \mathrm{eV}$ emission intensities.

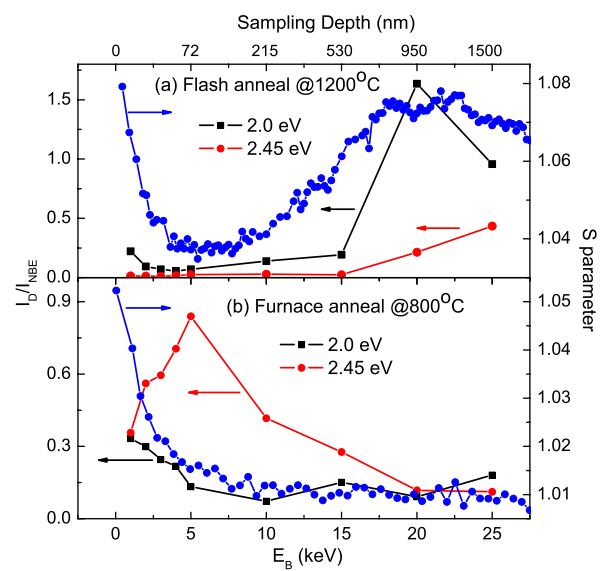

FIG. 2. (Color online) PAS and DRCLS defect densities vs. depth for Li-implanted $\mathrm{ZnO}$ after (a) flash and (b) furnace annealing. $V_{\mathrm{Zn}}$ densities and $I_{\mathrm{D}}(\sim 2.0 \mathrm{eV}) / I_{\mathrm{NBE}}$ correlate, in contrast to $I_{\mathrm{D}}(\sim 2.4 \mathrm{eV}) / I_{\mathrm{NBE}}$.

Figure 2 shows the correlation of DRCLS $I(\sim 2.0 \mathrm{eV})$ and $I(\sim 2.4 \mathrm{eV})$ with PAS $V_{\mathrm{Zn}}$ and vacancy cluster densities versus depth ${ }^{22}$ on the same Li-implanted $\mathrm{ZnO}$ crystals. In order to improve DRCLS depth resolution for higher $E_{\mathrm{B}}$, we employed a relatively simple subtraction method: we used Monte Carlo program CASINO (Ref. 27) to renormalize spectra from shallower layers for subtraction from deeper layer spectra. This procedure yields $I_{\mathrm{D}} / I_{\mathrm{NBE}}$ profiles with resolution comparable to the PAS $S$ parameter depth profiles. The $1200{ }^{\circ} \mathrm{C}$ flash-annealed $\mathrm{ZnO}$ in Fig. 2(a) displays a strong increase in $V_{\mathrm{Zn}}$ and vacancy cluster defects beginning at $\sim 100 \mathrm{~nm}$ and peaking at $\sim 1 \mu \mathrm{m}$. The latter corresponds to the depth of maximum implantation damage. $I(\sim 2.0) / I_{\mathrm{NBE}}$ also begins to increase at approximately the same depth, increases by approximately the same magnitude, and reaches a maximum at the same depth. By contrast, $I(\sim 2.4) / I_{\mathrm{NBE}}$ is low for depths of $500 \mathrm{~nm}$ or more, increasing only gradually for deeper excitation. The $800{ }^{\circ} \mathrm{C}$ furnace-annealed $\mathrm{ZnO}$ in Fig. 2(b) again shows a strong correlation between PAS $V_{\mathrm{Zn}}$-related defect densities and $I(\sim 2.0) / I_{\mathrm{NBE}}$, whereas $I(\sim 2.4) / I_{\mathrm{NBE}}$ exhibits a qualitatively different depth profile. Further evidence for this assignment includes near-surface (5 keV) DRCL spectra of $900{ }^{\circ} \mathrm{C}$ flash-annealed $\mathrm{ZnO}$ (not shown) that display over two orders of magnitude higher $I_{\mathrm{D}}(\sim 2 \mathrm{eV}) / I_{\mathrm{NBE}}$ and $I_{\mathrm{D}}(\sim 1.6 \mathrm{eV}) / I_{\mathrm{NBE}}$ (discussed below) compared with Fig. 1, in agreement with 2 orders of magnitude higher isolated $V_{\mathrm{Zn}}$ measured by PAS in this region. ${ }^{22}$ Note that the depth profile of $3.0 \mathrm{eV}$ emission as shown in Fig. 1(a) is not consistent with that of the PAS $S$ parameter. Hence, it may be due to higher order complex defects rather than $\mathrm{Zn}$ vacancies.

From the correlation of depth profiles in Fig. 2, the $\sim 2.0 \mathrm{eV}$ emission can be assigned to $\mathrm{Zn}$ vacancies or their complexes. This resolves the many contradictory assignments reported previously. ${ }^{6}$ Vanheusden $e t$ al. assigned the $2.45 \mathrm{eV}$ emissions to $\mathrm{O}$ vacancies. ${ }^{10}$ Even though PAS is not directly sensitive to $\mathrm{O}$ vacancies, our combined PAS-DRCLS results showing the completely different behavior of 2.4-2.5 $\mathrm{eV}$ vs the $\mathrm{Zn}$ vacancy emissions now demonstrate that optical emissions at energies typically assigned to $\mathrm{O}$ vacancies 


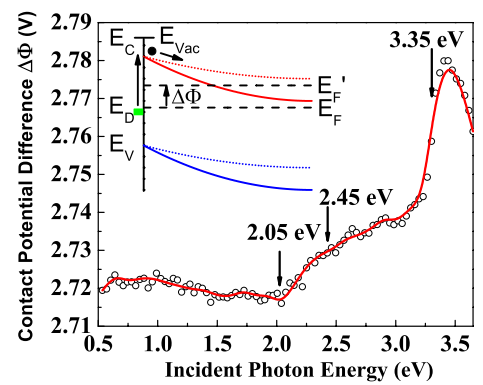

FIG. 3. (Color online) $\Delta \Phi$ vs $h v$ with onset of photodepopulation of deep levels $E_{\mathrm{D}}$ at $2.05 \mathrm{eV}$ below conduction band $E_{\mathrm{C}}$, photopopulation at $2.45 \mathrm{eV}$ above valence band $E_{\mathrm{V}}$, and band flattening at $h v>$ band gap $E_{\mathrm{G}}(3.35 \mathrm{eV})$.

are in fact unrelated to $\mathrm{Zn}$ vacancies. Indeed the spatial variations of the former that depend on specific annealing conditions also eliminate any role of bulk impurities. Both assignments are consistent with calculations showing $V_{\mathrm{Zn}}$ and $V_{\mathrm{O}}$ vacancies to be the most common native point defects, ${ }^{18}$ and both are the most commonly observed deep level features. Likewise, they agree with assignments based on metal-oxide and metal-eutectic reactions observed at $\mathrm{ZnO}$ Schottky barriers. ${ }^{16}$

SPS in a Kelvin probe (i.e., surface potential) force microscope (KPFM) shows that the $V_{\mathrm{Zn}}$-related DRCLS emission corresponds to optical transitions from the $\mathrm{ZnO}$ conduction band to gap states $2.1 \mathrm{eV}$ below. The SPS measurement consists of monitoring changes in surface electric potential with illumination as photon energy $h v$ sweeps from low to above band-gap values. The corresponding contact potential difference (CPD) between the surface and a reference probe changes as $h v$ exceeds threshold values for gap state population or depopulation. ${ }^{28}$ For $n$-type (upward) band bending and gap states of energy $E_{\mathrm{D}}$ located $E_{\mathrm{C}}-E_{\mathrm{D}}$ below the conduction band $E_{\mathrm{C}}$, Fig. 3 shows that photodepopulation removes negative surface charge and reduces the band bending, thereby raising the Fermi level $E_{\mathrm{F}}$ to $E_{\mathrm{F}}^{\prime}$ and lowering the surface potential $\Phi$ by $\Delta \Phi$. Here, $h v$ slope changes at $2.05,2.45$, and $3.35 \mathrm{eV}$ correspond to thresholds for electron photodepopulation, population, and free electron-hole pair transitions, respectively. These SPS features are characteristic of $\mathrm{ZnO}$ surfaces that exhibit luminescence peaks at these energies. Thus the $1.9-2.1 \mathrm{eV}$ peak in Fig. 1 corresponds to states $2.05 \mathrm{eV}$ below $E_{\mathrm{C}}$ while the $2.45 \mathrm{eV}$ peak corresponds to states $2.45 \mathrm{eV}$ above the valence band. This $2.05 \mathrm{eV}$ SPS feature is characteristic of $\mathrm{ZnO}$ surfaces for which surfacesensitive DRCLS exhibits strong 1.9-2.1 eV emission. Hence the $V_{\mathrm{Zn}}$-related defect luminescence emission at 1.9$2.1 \mathrm{eV}$ corresponds to an energy level at $\sim 2.05 \mathrm{eV}$ below the conduction band as revealed by SPS. This $0 /-1 V_{\mathrm{Zn}}$ transition energy is lower than the first-principles calculations of $\sim 2.7 \mathrm{eV}$ using a hybrid functional and finite-size corrections, ${ }^{21} 3.2 \mathrm{eV}$ using density-functional theory within the local-density approximation (LDA) and plane-wave pseudopotentials, ${ }^{5}$ and $3.8 \mathrm{eV}$ using the plane-wave pseudopotential total-energy and force method plus LDA. ${ }^{19}$

Combined DRCLS and PAS studies of implanted $\mathrm{ZnO}$ reveal that the 1.9-2.1 eV emissions in Fig. 1 correspond to large vacancy clusters (containing at least $3-4 V_{\mathrm{Zn}}$ ) and that

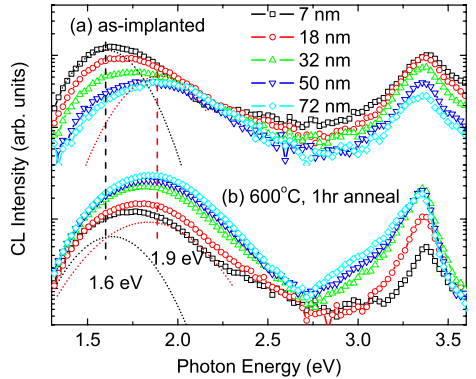

FIG. 4. (Color online) $70 \mathrm{~K} \mathrm{CL}$ spectra (1-5 keV) for (a) asreceived $\mathrm{N}$-implanted $\mathrm{ZnO}$ and after (b) $1 \mathrm{~h} 600{ }^{\circ} \mathrm{C}$ furnace annealing that induces $V_{\mathrm{Zn}}$ clustering. Dashed lines represent characteristic emissions at $\sim 1.6$ and $\sim 1.9 \mathrm{eV}$ as revealed by fitting.

the emission energies for small vacancy clusters $\left(\geq 2 \quad V_{\mathrm{Zn}}\right)$ are significantly lower. ${ }^{29}$ Previous positron experiments showed that small vacancy clusters are predominant in asimplanted $\mathrm{ZnO}$ while $600{ }^{\circ} \mathrm{C}$ furnace annealing induces coalescence into larger vacancy clusters and substantially $S$ parameter values. ${ }^{23}$ In Fig. 4(a), deep level emissions of the same $\mathrm{N}^{+}$-as-implanted crystals are deconvolved into peaks at 1.6 and $1.9 \mathrm{eV}$ with pronounced defect emission at $1.6 \mathrm{eV}$, in the near-surface $(7 \mathrm{~nm})$ region, shifting to $1.9 \mathrm{eV}$ at depths above $70 \mathrm{~nm}$. Electron paramagnetic resonance studies confirm the $\mathrm{Zn}$ vacancy nature of luminescence in this energy range. ${ }^{30}$ After the $600{ }^{\circ} \mathrm{C}$ anneal, Fig. 4(b) shows that the characteristic emission shifts to higher energy $(1.9 \mathrm{eV})$ and $I_{\mathrm{D}} / I_{\mathrm{NBE}}$ increases. Note the increasing defect energy with increasing depth (overall shift of the defect related emission with the probing depth in Fig. 4), indicating isolated or small cluster sizes near the free surface. A $1000{ }^{\circ} \mathrm{C}$ anneal dissociates the larger vacancy clusters, ${ }^{23}$ and the $1.9-2.1 \mathrm{eV}$ DRCLS feature decreases by nearly an order of magnitude with a corresponding increase in $\sim 2.4 \mathrm{eV} V_{\mathrm{O}}$-related emission (not shown). These results are consistent with vacancy cluster emission at 1.9-2.1 eV versus small vacancy cluster or isolated $V_{\mathrm{Zn}}$ emission at $\sim 1.6 \mathrm{eV}$. They indicate that large vacancy clusters lie $>0.3 \mathrm{eV}$ lower in the $\mathrm{ZnO}$ band gap and are the predominant defect responsible for $\sim 2 \mathrm{eV}$ "red" photoluminescence.

Figure 4 also provides a calibration of DRCLS with vacancy concentrations obtained with positrons. The $\mathrm{N}^{+}$-as-implanted $\mathrm{ZnO}$ contains an estimated concentration of small vacancy clusters [denoted $\left(V_{\mathrm{Zn}}\right)_{\mathrm{n}}$ ] of 1-2 $\times 10^{18} \mathrm{~cm}^{-3},{ }^{23}$ corresponding to $I(\sim 2.0 \mathrm{eV}) / I_{\mathrm{NBE}} \sim 1$. Since $I(\sim 2.0 \mathrm{eV}) / I_{\mathrm{NBE}} \sim 1.6$ in Fig. 2 (a) at the peak implantation depth, then $\left(V_{\mathrm{Zn}}\right)_{\mathrm{n}} \sim 1.6 \times 10^{18} \mathrm{~cm}^{-3}$, in line with previous estimates. ${ }^{22}$ Calibration at this depth permits estimates of $\left(V_{\mathrm{Zn}}\right)_{\mathrm{n}}$ concentration much closer to the surface than PAS conventionally permits.

The relative densities of large and small vacancy clusters or isolated $V_{\mathrm{Zn}}$, together with $V_{\mathrm{O}}$, and all as a function of depth account for the ZnO's local resistance self-consistently. $\mathrm{Zn}$ vacancies and vacancy clusters play different roles electrically. $V_{\mathrm{Zn}}$ defects act as compensating acceptors and increase resistance, while vacancy clusters remove isolated $V_{\mathrm{Zn}}$ and/or deactivate Li dopants, thereby decreasing resistance, as observed previously in experiments with irradiationinduced electrical isolation. ${ }^{31}$ As Fig. 2 showed for $\mathrm{Li}^{-}$ 


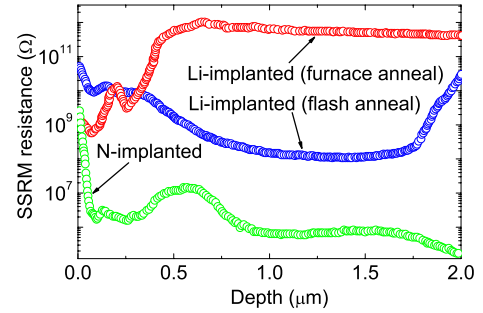

FIG. 5. (Color online) SSRM resistance depth profiles of Li- and $\mathrm{N}$-implanted $\mathrm{ZnO}$ in Figs. 2 and 4, respectively.

implanted samples, $1200{ }^{\circ} \mathrm{C}$ flash anneal generates a high concentration $\left(10^{18}-10^{20} \mathrm{~cm}^{-3}\right)$ of vacancy clusters in the $500-1500 \mathrm{~nm}$ region. These vacancy clusters reduce densities of isolated $V_{\mathrm{Zn}}$, small vacancy clusters, and uncomplexed $\mathrm{Li}$, all of which reduce the compensating acceptor density. Accordingly, the scanning spreading resistance microscopy (SSRM) resistance profile of this crystal in Fig. 5 displays a major (three orders of magnitude) decrease within the same region. As Fig. 4 showed, N-implantation introduces isolated $\mathrm{Zn}$ vacancies in the intimate surface region $(<50 \mathrm{~nm})$. The corresponding surface resistance in Fig. 5 increased by over three orders of magnitude. ${ }^{32}$

In general, both $V_{\mathrm{Zn}}$ and $V_{\mathrm{O}}$ densities are needed to account for resistance in $\mathrm{ZnO}$ self-consistently. The $\sim 2.4 \mathrm{eV}$ emission peak attributed to oxygen vacancies acting as deep donors exhibits a pronounced maximum at $\sim 70 \mathrm{~nm}$ depth for $800{ }^{\circ} \mathrm{C}$ furnace-annealed $\mathrm{ZnO}$ in Fig. 2. This $2.4 \mathrm{eV}$ peak maximum can account for the sharp drop in SSRM resistance at the same depth for this crystal in Fig. 5, whereas the $\sim 2.0 \mathrm{eV}$ peak intensity is low and varying slowly at these depths. Thus the combination of low concentration $V_{\mathrm{Zn}}$ and vacancy clusters plus elevated $V_{\mathrm{O}}$ in the near-surface $(<500 \mathrm{~nm})$ region act to decrease surface resistance by nearly four orders of magnitude relative to the bulk, the lowest near-surface resistance of all Li-implanted $\mathrm{ZnO}$ studied. Other recent $S$ parameter correlations with optical/transport properties include $\mathrm{ZnO},{ }^{22,23,31} \mathrm{GaN}^{33}$ and $\mathrm{InN}$ (Ref. 34) since the $S$ parameter reflects the vacancy content, which undoubtedly affect the optoelectronic properties of semiconductors.

In summary, combined PAS, DRCLS, and SPS studies reveal the $V_{\mathrm{Zn}}$ defect nature of optical emissions in the range of $1.6-2.1 \mathrm{eV}$, the energy-level position of vacancy clusters at $1.9-2.1 \mathrm{eV}$ below the conduction band, and the energylevel position of isolated $V_{\mathrm{Zn}}$ defects or small clusters $0.3 \mathrm{eV}$ higher above the valence band. DRCLS-measured vacancy cluster and $V_{\mathrm{O}}$-related emissions combined with SSRM resistance within the same near-surface regions reveal the different compensating nature of vacancy clusters on $\mathrm{ZnO}$ carrier concentration and the competing roles of $V_{\mathrm{Zn}}$ and $V_{\mathrm{O}}$ defects on $\mathrm{ZnO}$ resistance. These results resolve the contradictory energetic assignments for $V_{\mathrm{Zn}}$ and add weight to the $V_{\mathrm{O}}$-related defect assignment reported previously. Furthermore, these combined results confirm the acceptor-versus donorlike behavior associated with these two optical emissions and demonstrate their utility. The physical nature of the defects that dominate optical features of this widely studied semiconductor and the consistent explanation for ZnO's effective free-carrier densities on a local scale enable a deeper understanding of many $\mathrm{ZnO}$ properties and their applications.

The authors gratefully acknowledge support from the National Science Foundation Grant No. DMR-0513968 (Verne Hess) and the Norwegian Research Council through the NANOMAT and FRINAT programs. F.T. acknowledges the support from the Academy of Finland. *dong.70@ osu.edu

${ }^{1}$ D. C. Look, Mater. Sci. Eng., B 80, 383 (2001).

${ }^{2}$ S. J. Pearton et al., Prog. Mater. Sci. 50, 293 (2005).

${ }^{3}$ D. C. Look, J. Electron. Mater. 35, 1299 (2006).

${ }^{4}$ Ü. Özgür et al., J. Appl. Phys. 98, 041301 (2005).

${ }^{5}$ A. Janotti and C. G. Van de Walle, Phys. Rev. B 76, 165202 (2007).

${ }^{6}$ M. D. McCluskey and S. J. Jokela, J. Appl. Phys. 106, 071101 (2009).

${ }^{7}$ D. C. Look et al., Phys. Rev. Lett. 95, 225502 (2005).

${ }^{8}$ F. A. Selim et al., Phys. Rev. Lett. 99, 085502 (2007).

${ }^{9}$ C. H. Ahn et al., J. Appl. Phys. 105, 013502 (2009).

${ }^{10}$ K. Vanheusden et al., Appl. Phys. Lett. 68, 403 (1996).

${ }^{11}$ Y. W. Heo et al., J. Appl. Phys. 98, 073502 (2005).

${ }^{12}$ M. A. Reshchikov et al., J. Appl. Phys. 103, 103514 (2008).

${ }^{13}$ Q. X. Zhao et al., Appl. Phys. Lett. 87, 211912 (2005).

${ }^{14}$ T. M. Børseth et al., Appl. Phys. Lett. 89, 262112 (2006).

${ }^{15}$ L. J. Brillson, J. Vac. Sci. Technol. B 19, 1762 (2001).

${ }^{16}$ L. J. Brillson et al., Appl. Phys. Lett. 90, 102116 (2007).

${ }^{17}$ S. Lany and A. Zunger, Phys. Rev. B 78, 235104 (2008).

${ }^{18}$ A. F. Kohan et al., Phys. Rev. B 61, 15019 (2000).

${ }^{19}$ S. B. Zhang et al., Phys. Rev. B 63, 075205 (2001).

${ }^{20}$ P. Erhart et al., Phys. Rev. B 73, 205203 (2006).
${ }^{21}$ F. Oba et al., Phys. Rev. B 77, 245202 (2008).

${ }^{22}$ T. Moe Børseth et al., Phys. Rev. B 74, 161202(R) (2006).

${ }^{23}$ T. M. Børseth et al., Phys. Rev. B 77, 045204 (2008).

${ }^{24}$ Z. Q. Chen et al., Phys. Rev. B 71, 115213 (2005).

${ }^{25}$ F. Tuomisto et al., Phys. Rev. Lett. 91, 205502 (2003).

${ }^{26}$ H. C. Ong et al., Appl. Phys. Lett. 78, 2667 (2001).

${ }^{27}$ D. Drouin et al., Scanning 29, 92 (2007).

${ }^{28}$ L. Kronik and Y. Shapira, Surf. Sci. Rep. 37, 1 (1999).

${ }^{29}$ Note that these clusters contain also O vacancies as otherwise the positron data would be similar to isolated $V_{\mathrm{Zn}}$ (for the data to be different as in this case, a larger connected open volume is needed).

${ }^{30}$ L. A. Kappers et al., Nucl. Instrum. Methods Phys. Res. B 266, 2953 (2008).

${ }^{31}$ A. Zubiaga et al., Phys. Rev. B 78, 035125 (2008).

${ }^{32}$ Note: any variations in surface roughness at the outer surface of the cross-sectional profile cuts could perturb outer surface SSRM values; however, these do not mask the overall subsurface and bulk systematics.

${ }^{33}$ F. Tuomisto et al., Appl. Phys. Lett. 90, 121915 (2007).

${ }^{34}$ F. Tuomisto, A. Pelli, K. M. Yu, W. Walukiewicz, and W. J. Schaff, Phys. Rev. B 75, 193201 (2007). 